\title{
ON SIMILARITY IN THE BEHAVIOR OF SODIUM
}

\section{AND POTASSIUM}

W. J. V. OSTERHOUT

(WITH FOUR FIGURES)

It is commonly mentioned by textbooks, as worthy of remark, that sodium and potassium agree closely in chemical behavior, but differ fundamentally in their effects upon plants.

This general statement is founded on the study of the nutritive functions of sodium and potassium. There is no a-priori reason for supposing it to be true in the field of toxic or of protective action. As this is a point of general interest I have made some experiments with reference to it.

Two extensive series of experiments, one on sodium, the other on potassium, were carried on simultaneously. They were found to show a remarkable degree of agreement in the action of these two substances.

The experiments relating to sodium have already been described, ${ }^{\mathrm{x}}$ while those on potassium have been withheld from publication, pending thecompletion of further observations on the mutually antagonistic action of sodium and potassium.

Most of the experiments were made with a variety of wheat known as Early Genesee. The technique has been fully described in a previous paper. $^{2}$

\section{TOXIC ACTION}

In the earliest studies which I made on balanced solutions, I was struck with the fact that $\mathrm{Na}$ and $\mathrm{K}$ agree closely in their toxic effect on plants.

These results I have found to hold in an extensive series of experiments, including algae, liverworts, Equisetum, and some thirteen genera of flowering plants. While there are doubtless some exceptions, the general rule seems to be that $\mathrm{Na}$ and $\mathrm{K}$ are closely similar in their toxic action.

I Jahrb. Wiss. Bot. 46: 121. I908.

2 Bot. Gazette 44:266. I907.

Botanical Gazette, vol. 48] 
The most careful quantitative studies which we possess on this point are those of Miss Magowan. ${ }^{3}$ These studies show that the toxicity curves for $\mathrm{K}$ and $\mathrm{Na}$ are practically identical, while the corresponding curve for $\mathrm{Mg}$ shows a much higher, and that for $\mathrm{Ca}$ a much lower degree of toxicity.

\section{ANTAGONISTIC ACTION 4}

The antagonistic action of monovalent kations on each other has especial interest in view of the experiments of LOEB $^{5}$ on Fundulus, which offer a certain analogy with those of LINDER and Picton. ${ }^{6}$ In these experiments monovalent kations antagonized bivalent but did not antagonize other monovalent kations.

The curve of antagonism between $\mathrm{NaCl}$ and $\mathrm{KCl}$ shows two maxima. The location of these maxima, however, is not constant, but varies somewhat in different series of experiments. Table I and fig. $I$ show the average result of four series. The antagonism

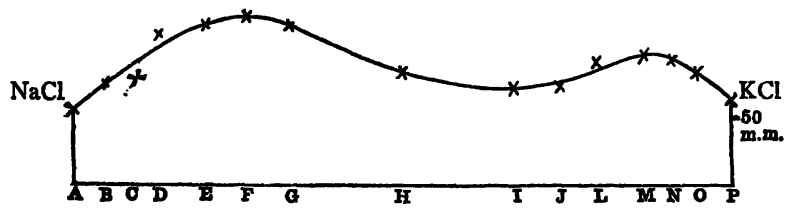

FIG. I.-Antagonism curve, $\mathrm{NaCl}$ vs. $\mathrm{KCl}$. The ordinates represent millimeters of growth of the roots of wheat. The ordinate at $\mathrm{A}$ represents the growth in pure $\mathrm{NaCl}$, that at $\mathrm{P}$ the growth in pure $\mathrm{KCl}$. The other ordinates represent growth in mixtures of $\mathrm{NaCl}$ and $\mathrm{KCl}$, the proportions of which are found opposite the corresponding letters in table $\mathrm{I}$ : thus the ordinate at $\mathrm{H}$ represents growth in a mixture of roocc $\mathrm{NaCl}+$ roocc $\mathrm{KCl}$.

is comparatively slight. I have also noticed antagonism between $\mathrm{Na}$ and $\mathrm{K}$ in liverworts.

Table II and fig. 2 show that both $\mathrm{Na}$ and $\mathrm{K}$ antagonize $\mathrm{NH}_{4}$, and that their effects are very similar.

3 Ibid., 45:45. Igo8.

4 Cf. facts and literature given by Kearney and Cameron, Rept. No. 7 I, U. S. Dept. Agr. I902; and by BENECKE, Ber. Deutsch. Bot. Gesells. 25:322. I907.

5 American Journal of Physiology 3:327. I900.

6 Cf. Hober und Gordon, Hofmeister's Beitr. Chem. Physiol. und Pathol. 5:432. I904. 
TABLE I

WheAT (GROWTH DURING 30 DAYS). ALl QUANTITIES GIVEN ARE CUBIC CENTIMETERS OF $0.12 m$ SOLUTIONS

\begin{tabular}{|c|c|c|}
\hline Culture solution & $\begin{array}{l}\text { Corresponding } \\
\text { point on } \\
\text { curve (fig. I) }\end{array}$ & $\begin{array}{l}\text { Aggregate length } \\
\text { of ronts per plant } \\
\text { : } \mathrm{n} \mathrm{mm}\end{array}$ \\
\hline $\mathrm{NaCl}$. & A & 55 \\
\hline $\begin{array}{rl}\mathrm{r} 00 \mathrm{NaCl} & \mathrm{KCl} \\
5 & \mathrm{KC}\end{array}$ & B & 75 \\
\hline roo $\begin{array}{rl}\mathrm{NaCl} & \mathrm{KCl}\end{array}$, $\ldots \ldots \ldots$ & $\mathrm{C}$ & 80 \\
\hline $\begin{aligned} & \text { Ioo } \mathrm{NaCl} \\
& \text { I5 } \mathrm{KCl}\end{aligned}$ & $\mathrm{D}$ & II 5 \\
\hline $\left.\begin{array}{rl}\text { roo } & \mathrm{NaCl} \\
25 \mathrm{KCl}\end{array}\right\}$ & E & I 20 \\
\hline $\left.\begin{array}{rl}100 & \mathrm{NaCl} \\
35 & \mathrm{KCl}\end{array}\right\} \ldots \ldots \ldots$ & $\mathbf{F}$ & 130 \\
\hline $\begin{array}{rl}100 & \mathrm{NaCl} \\
50 & \mathrm{KCl}\end{array} \ldots \ldots \ldots$ & G & I 2 I \\
\hline $\begin{array}{l}\text { Ioo } \mathrm{NaCl}\} \\
\text { roo } \mathrm{KCl}\}\end{array}$ & $\mathrm{H}$ & 85 \\
\hline $\left.\begin{array}{r}50 \mathrm{NaCl} \\
\text { roo } \mathrm{KCl}\end{array}\right\}$. & I & 75 \\
\hline $\left.\begin{array}{rl}35 & \mathrm{NaCl} \\
\text { roo } & \mathrm{KCl}\end{array}\right\}$ & $J$ & 80 \\
\hline $\left.\begin{array}{rl}25 & \mathrm{NaCl} \\
100 \mathrm{KCl}\end{array}\right\}$ & L & 95 \\
\hline $\begin{array}{r}\text { I5 } \mathrm{NaCl}) \\
\text { Ioo } \mathrm{KCl} ; \ldots . .\end{array}$ & M & 100 \\
\hline $\left.\begin{array}{r}\text { Io } \mathrm{NaCl} \\
\text { Ioo } \mathrm{KCl}\end{array}\right\}$ & $\mathrm{N}$ & 95 \\
\hline $\left.\begin{array}{rl}5 & \mathrm{NaCl} \\
\mathrm{KCl}\end{array}\right\}$ & $\mathrm{O}$ & 85 \\
\hline $\mathrm{KCl} \ldots$ & $\mathrm{P}$ & 65 \\
\hline
\end{tabular}

Distilled water, $725^{\mathrm{mm}}$

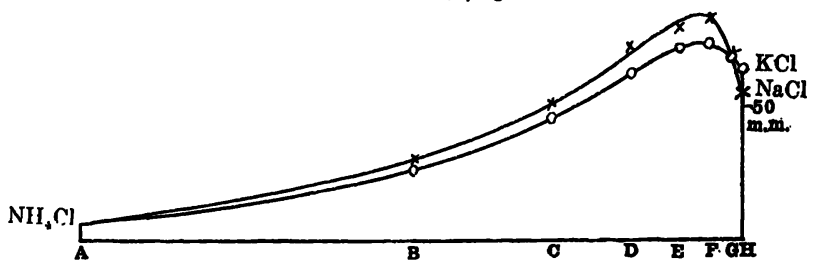

Fig. 2.-Antagonism curve, $\mathrm{NH}_{4} \mathrm{Cl}$ vs. $\mathrm{NaCl}$ (upper curve -x-x-x-) and $\mathrm{NH}_{4} \mathrm{Cl}$ vs. $\mathrm{KCl}$ (lower curve -o-o-o-). Each ordinate represents the amount of growth of wheat roots in a solution whose composition is given opposite the corresponding letter in table II. 
TABLE II

Wheat (GRowth DURING 30 DAYS). All QUANTITIES GIVEN ARE CUBIC CENTIMETERS OF $0.12 m$ SOLUTIONS

\begin{tabular}{|c|c|c|c|c|}
\hline Culture solution & $\begin{array}{c}\text { Aggregate } \\
\text { length of roots } \\
\text { per plant in } \mathrm{mm}\end{array}$ & $\begin{array}{l}\text { Corresponding } \\
\text { point on curve } \\
\text { (fig. 2) }\end{array}$ & Culture solution & $\begin{array}{l}\text { Aggregate length } \\
\text { of roots per } \\
\text { plant in } \mathrm{mm}\end{array}$ \\
\hline $\mathrm{NHC}_{4} \mathrm{l} . \ldots \ldots$ & 6.2 & A & $\mathrm{NH}_{4} \mathrm{Cl}$. & 6.2 \\
\hline $\left.\begin{array}{l}\text { Ioo } \mathrm{NH}_{4} \mathrm{Cl} \\
\text { I00 } \mathrm{NaCl}\end{array}\right\} \ldots$ & $3 \mathrm{I}$ & B & $\left.\begin{array}{ll}\text { Ioo } & \mathrm{NH}_{4} \mathrm{Cl} \\
\text { I0o } & \mathrm{KCl}\end{array}\right\}$ & $27 \cdot 5$ \\
\hline $\left.\begin{array}{r}40 \mathrm{NH}_{4} \mathrm{Cl} \\
\text { I00 } \mathrm{NaCl}\end{array}\right\}$. & $5^{2}$ & C. & $\left\{\begin{array}{rl}40 & \mathrm{NH}_{4} \mathrm{Cl} \\
100 & \mathrm{KCl}\end{array}\right\}$ & 46 \\
\hline $\left.\begin{array}{r}20 \mathrm{NH}_{4} \mathrm{Cl} \\
100 \mathrm{NaCl}\end{array}\right\}$ & 74 & $\mathrm{D}$ & $\left.\begin{array}{rl}20 & \mathrm{NH}_{4} \mathrm{Cl} \\
100 \mathrm{KCl}\end{array}\right\}$ & 62 \\
\hline $\left.\begin{array}{l}\text { Io } \mathrm{NH}_{4} \mathrm{Cl} \\
\text { roo } \mathrm{NaCl}\end{array}\right\}$. & 80 & $\mathbf{E}$ & $\left.\begin{array}{l}\text { Io } \mathrm{NH}_{4} \mathrm{Cl} \\
\text { I00 } \mathrm{KCl}\end{array}\right\}$ & $72 \cdot 5$ \\
\hline $\left.\begin{array}{r}5 \mathrm{NH}_{4} \mathrm{Cl} \\
\text { I0o } \mathrm{NaCl}\end{array}\right\} \ldots$ & 85 & $F$ & $\left\{\begin{array}{rl}5 & \mathrm{NH}_{4} \mathrm{Cl} \\
100 & \mathrm{KCl}\end{array}\right\}$ & 75 \\
\hline $\left.\begin{array}{r}\mathrm{r} \mathrm{NH}_{4} \mathrm{Cl} \\
\text { I00 } \mathrm{NaCl}\end{array}\right\}$. & 67 & G & 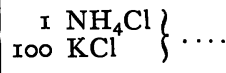 & 68 \\
\hline $\mathrm{NaCl} . . .$. & 55 & $\mathrm{H}$ & $\mathrm{KCl}$. & 66 \\
\hline
\end{tabular}

Distilled water, $725 \mathrm{~mm}$

Experiments with magnesium show that it is antagonized in about the same degree by both $\mathrm{Na}$ and $\mathrm{K}$ (table III and fig. 3).

FIG. 3.-Antagonism curve, $\mathrm{MgCl}_{2}$ vs. $\mathrm{NaCl}$ (upper curve $-\mathbf{x}-\mathbf{x}-\mathbf{x}-$ ) and $\mathrm{MgCl}_{2}$ vs. $\mathrm{KCl}$ (lower curve -o-o-o-. Each ordinate represents the amount of growth of wheat roots in a solution whose composition is given opposite the corresponding letter in table III.

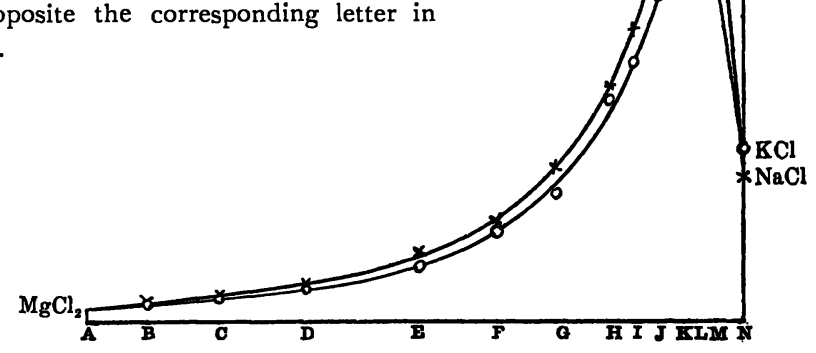


TABLE III

Wheat (GROWTH DURING 30 DAYS). ALL QUANTITIES GIVEN ARE CUBIC CENTIMETERS OF O.I $2 m$ SOLUTIONS

\begin{tabular}{|c|c|c|c|c|}
\hline Culture solution & $\begin{array}{c}\text { Aggregate length } \\
\text { of roots per } \\
\text { plant in } \mathrm{mm}\end{array}$ & $\begin{array}{l}\text { Corresponding } \\
\text { point on curve } \\
\text { (fig. 3) }\end{array}$ & Culture solution & $\begin{array}{l}\text { Aggregate length } \\
\text { of roots per } \\
\text { plant in } \mathrm{mm}\end{array}$ \\
\hline $\mathrm{MgCl}_{2} \ldots \ldots \ldots$ & 5 & A & $\mathrm{MgCl}_{2} \ldots \ldots$ & 5 \\
\hline $\left.\begin{array}{rl}\text { Io } & \mathrm{MgCl}_{2} \\
\text { Io } & \mathrm{NaCl}\end{array}\right\}$ & $7 \cdot 5$ & B & $\left.\begin{array}{l}\text { I00 } \mathrm{MgCl}_{2} \\
\text { Io } \mathrm{KCl}\end{array}\right\}$ & $7 \cdot 5$ \\
\hline $\left.\begin{array}{rl}\text { I00 } & \mathrm{MgCl}_{2} \\
25 & \mathrm{NaCl}\end{array}\right\} \ldots \ldots$. & IO & C & $\left.\begin{array}{r}100 \mathrm{MgCl}_{2} \\
25 \mathrm{KCl}\end{array}\right\}$ & 8.7 \\
\hline $\left.\begin{array}{rl}\text { IoO } & \mathrm{MgCl}_{2} \\
50 & \mathrm{NaCl}\end{array}\right\}$ & I3.7 & $\mathrm{D}$ & $\begin{array}{c}\text { Ioo } \mathrm{MgCl}_{2} \\
50 \mathrm{KCl}\end{array}$ & 12.5 \\
\hline $\left.\begin{array}{l}\text { I00 } \mathrm{MgCl}_{2} \\
\text { I00 } \mathrm{NaCl}\end{array}\right\}$ & 25 & $\mathrm{E}$ & $\left.\begin{array}{l}100 \mathrm{MgCl}_{2} \\
100 \mathrm{KCl}\end{array}\right\}$ & $2 I$ \\
\hline $\begin{aligned}\left.\begin{array}{rl}60 & \mathrm{MgCl}_{2} \\
\text { 100 } & \mathrm{NaCl}\end{array}\right\}\end{aligned}$ & $37 \cdot 5$ & $\mathrm{~F}$ & $\left.\begin{array}{l}60 \mathrm{MgCl}_{2} \\
\text { 100 } \mathrm{KCl}\end{array}\right\}$ & 34 \\
\hline $\left.\begin{array}{rl}40 \mathrm{MgCl}_{2} \\
\text { I00 } \mathrm{NaCl}\end{array}\right\}$ & 50.7 & G & $\left.\begin{array}{r}40 \mathrm{MgCl}_{2} \\
\text { 100 KCl}\end{array}\right\}$ & 48.7 \\
\hline $\left.\begin{array}{rl}25 & \mathrm{MgCl}_{2} \\
100 & \mathrm{NaCl}\end{array}\right\}$ & 90 & $\mathrm{H}$ & $\left.\begin{array}{r}25 \mathrm{MgCl}_{2} \\
\text { 100 } \mathrm{KCl}\end{array}\right\}$ & 84 \\
\hline $\left.\begin{array}{r}20 \mathrm{MgCl}_{2} \\
\text { 100 } \mathrm{NaCl}\end{array}\right\}$ & II 2 & I & $\left.\begin{array}{r}20 \mathrm{MgCl}_{2} \\
100 \mathrm{KCl}\end{array}\right\}$ & 99 \\
\hline $\left.\begin{array}{rl}\text { I5 } & \mathrm{MgCl}_{2} \\
\text { I0O } & \mathrm{NaCl}\end{array}\right\}$ & I4O & $\mathrm{J}$ & $\left.\begin{array}{l}\text { I5 } \mathrm{MgCl}_{2} \\
\text { I00 } \mathrm{KCl}\end{array}\right\}$ & 125 \\
\hline $\left.\begin{array}{l}\text { Io } \mathrm{MgCl}_{2} \\
\text { I0० } \mathrm{NaCl}\end{array}\right\}$. & $\mathrm{I}_{7} 6$ & $\mathrm{~K}$ & $\left.\begin{array}{l}\text { Io } \mathrm{MgCl}_{2} \\
\text { I00 } \mathrm{KCl}\end{array}\right\}$ & $\mathrm{I}^{2}$ \\
\hline $\left.\begin{array}{ll}7.5 & \mathrm{MgCl}_{2} \\
\text { I00 } & \mathrm{NaCl}\end{array}\right\} \ldots \ldots$ & 210 & $\mathrm{~L}$ & $\left.\begin{array}{l}7.5 \mathrm{MgCl}_{2} \\
100 \mathrm{KCl}\end{array}\right\}$. & I7 I \\
\hline $\left.\begin{array}{rl}5 & \mathrm{MgCl}_{2} \\
\text { I0० } & \mathrm{NaCl}\end{array}\right\}$. & I72 & $\mathbf{M}$ & $\left.\begin{array}{r}5 \mathrm{MgCl}_{2} \\
\text { 100 } \mathrm{KCl}\end{array}\right\}$. & I35 \\
\hline $\mathrm{NaCl} \ldots \ldots$ & 55 & $\mathrm{~N}$ & $\mathrm{NaCl} .$. & 66 \\
\hline
\end{tabular}

Distilled water, $725 \mathrm{~mm}$

In algae $\mathrm{I}$ have found that $\mathrm{MgCl}_{2}$ is much more strikingly antagonized by $\mathrm{KCl}$ than by $\mathrm{NaCl}$.

The experiments with calcium show a more marked antagonism than any of the other cases. We find that $\mathrm{Ca}$ is antagonized to a slightly greater degree by $\mathrm{K}$ than by $\mathrm{Na}$ (table IV and fig. 4).

Similar results were obtained with algae, liverworts, Equisetum, and some fifteen genera of flowering plants.

From the facts here set forth it is clear that in their toxic and 
protective effects sodium and potassium show great similarity. As this does not seem to be the case in the field of nutritive effects, we

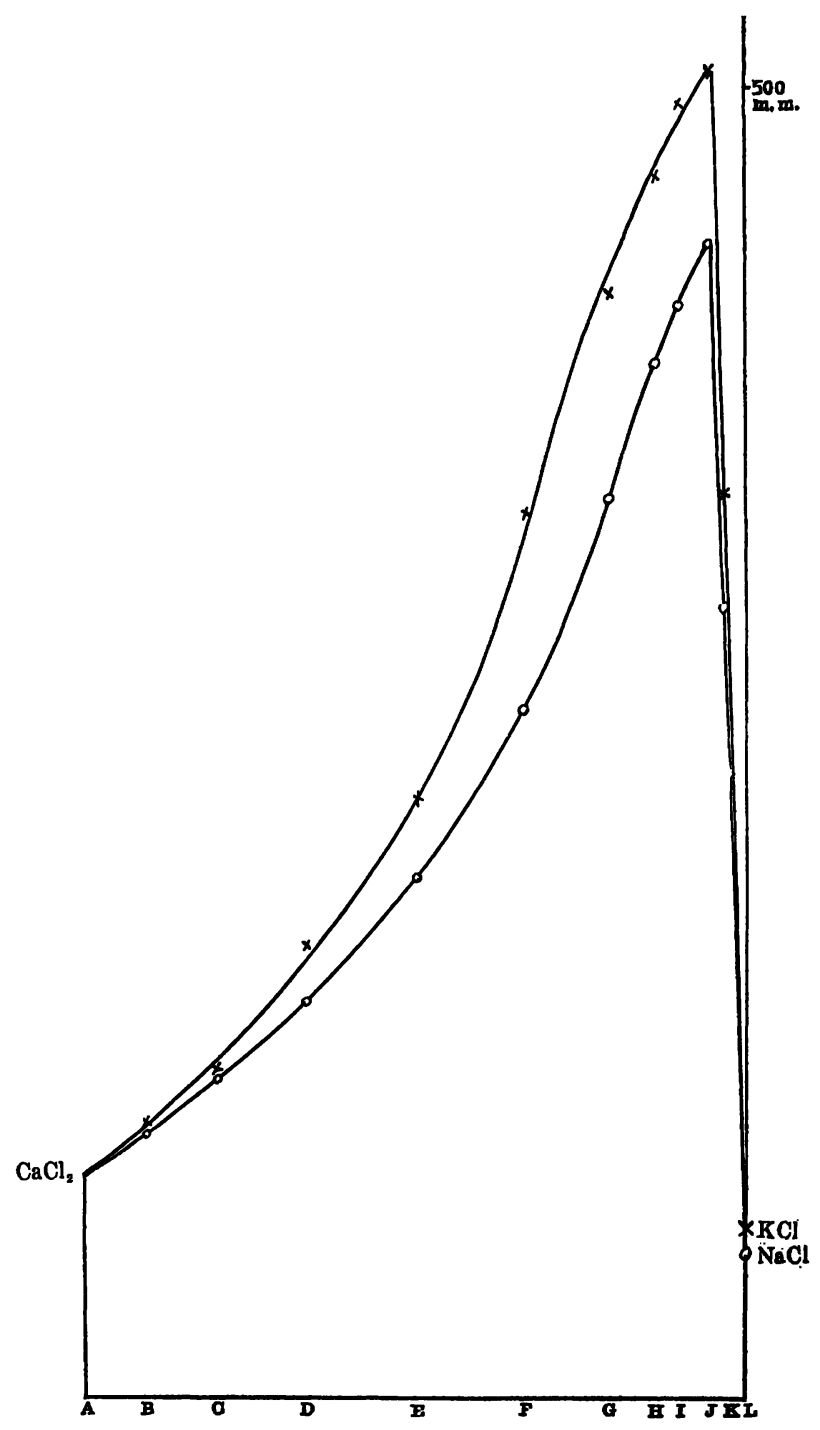

Fig. 4.-Antagonism curve, $\mathrm{CaCl}_{2}$ vs. $\mathrm{KCl}$ (upper curve $-\mathrm{x}-\mathrm{x}-\mathrm{x}-$ ) and $\mathrm{CaCl}_{2}$ vs. $\mathrm{NaCl}$ (lower curve $0-0-0$ ). Each ordinate represents the amount of growth of wheat roots in a solution whose composition is given opposite the corresponding letter in table IV 
seem to have in this case a means of distinguishing clearly between nutritive and protective action.

TABLE IV

Wheat (GROWTH DURING 30 DAYS). All QUANTIties given are cubic CENTIMETERS OF O.I $2 m$ SOLUTIONS

\begin{tabular}{|c|c|c|c|c|}
\hline Culture solution & $\left|\begin{array}{c}\text { Aggregate length } \\
\text { of roots per } \\
\text { plant in } \mathrm{mm}\end{array}\right|$ & $\begin{array}{l}\text { Corresponding } \\
\text { point on curve } \\
(\text { fig. } 4)\end{array}$ & Culture solution & $\begin{array}{l}\text { Aggregate length } \\
\text { of roots per } \\
\text { plant in } \mathrm{mm}\end{array}$ \\
\hline $\mathrm{CaCl}_{2}$. & 85 & A & $\mathrm{CaCl}_{2}$ & 85 \\
\hline $\left.\begin{array}{r}\text { Ioo } \mathrm{CaCl}_{2} \\
\text { Io } \mathrm{NaCl}\end{array}\right\}$ & I00 & B & $\left.\begin{array}{r}\text { Ioo } \mathrm{CaCl}_{2} \\
\text { Io } \mathrm{KCl}\end{array}\right\}$ & 105 \\
\hline $\left.\begin{array}{r}\text { 100 } \mathrm{CaCl}_{2} \\
25 \mathrm{NaCl}\end{array}\right\}$ & II7 & $\mathrm{C}$ & $\left.\begin{array}{rl}\text { I00 } & \mathrm{CaCl}_{2} \\
25 & \mathrm{KCl}\end{array}\right\}$ & I 25 \\
\hline $\left.\begin{array}{r}\text { 100 } \mathrm{CaCl}_{2} \\
50 \mathrm{NaCl}\end{array}\right\}$ & I 50 & $\mathrm{D}$ & $\left.\begin{array}{r}\text { I00 } \mathrm{CaCl}_{2} \\
50 \mathrm{KCl}^{\prime}\end{array}\right\}$ & I74 \\
\hline $\left.\begin{array}{l}\text { I00 } \mathrm{CaCl}_{2} \\
\text { 100 } \mathrm{NaCl}\end{array}\right\}$ & r98 & $\mathrm{E}$ & $\left.\begin{array}{l}\text { Ioo } \mathrm{CaCl}_{2} \\
\text { 100 } \mathrm{KCl}\end{array}\right\}$ & 230 \\
\hline $\left.\begin{array}{r}50 \mathrm{CaCl}_{2} \\
\text { 100 } \mathrm{NaCl}\end{array}\right\} \ldots$ & 262 & $\mathrm{~F}$ & $\left.\begin{array}{r}50 \mathrm{CaCl}_{2} \\
\text { 100 } \mathrm{KCl}\end{array}\right\}$ & 337 \\
\hline $\left.\begin{array}{r}25 \mathrm{CaCl}_{2} \\
\text { 100 } \mathrm{NaCl}\end{array}\right\}$ & 342 & G & $\left.\begin{array}{r}25 \mathrm{CaCl}_{2} \\
100 \mathrm{KCl}\end{array}\right\}$ & 420 \\
\hline $\left.\begin{array}{r}\text { I5 } \mathrm{CaCl}_{2} \\
\text { I00 } \mathrm{NaCl}\end{array}\right\}$ & 390 & $\mathrm{H}$ & $\left.\begin{array}{r}\text { r5 } \mathrm{CaCl}_{2} \\
100 \mathrm{KCl}\end{array}\right\}$ & 464 \\
\hline $\left.\begin{array}{r}\text { Io } \mathrm{CaCl}_{2} \\
\text { I } 00 \mathrm{NaCl}\end{array}\right\}$ & $4 \mathrm{I} 6$ & I & $\left.\begin{array}{r}\text { Io } \mathrm{CaCl}_{2} \\
\text { 100 } \mathrm{KCl}\end{array}\right\}$ & 492 \\
\hline $\left.\begin{array}{r}5 \mathrm{CaCl}_{2} \\
\text { I } 00 \mathrm{NaCl}\end{array}\right\}$ & 440 & $\mathrm{~J}$ & $\left.\begin{array}{r}5 \mathrm{CaCl}_{2} \\
\text { 100 } \mathrm{KCl}\end{array}\right\}$. & $5 \circ 7$ \\
\hline $\left.\begin{array}{r}\text { I } \mathrm{CaCl}_{2} \\
\text { I00 } \mathrm{NaCl}\end{array}\right\}$ & 300 & $\mathrm{~K}$ & $\left.\begin{array}{r}\text { I } \mathrm{CaCl}_{2} \\
\text { 100 } \mathrm{KCl}\end{array}\right\}$. & 346 \\
\hline $\mathrm{NaCl}$. . & 55 & L & $\mathrm{KCl}$. & 66 \\
\hline
\end{tabular}

Distilled water, $725 \mathrm{~mm}$

\section{SUMMARY}

The accepted idea that sodium and potassium have entirely different effects upon plants is not valid in the field of toxic and protective action. Here their behavior shows the close similarity which their near chemical relationship would lead us to expect.

University of CaLIFornia

BERKELEY 